\title{
In-Beam Gamma Ray Spectroscopy of ${ }^{58} \mathrm{Co}$
}

\author{
M. A. G. Silveira, N. H. Medina, J. R. B. Oliveira, J. A. Alcântara-Nuñez, E. W. \\ Cybulska, H. Dias, M. N. Rao, R. V. Ribas, W. A. Seale, and K. T. Wiedemann \\ Instituto de Física, Universidade de São Paulo, São Paulo, SP, Brazil
}

Received on 12 November, 2005

\begin{abstract}
The odd-odd ${ }^{58} \mathrm{Co}$ nucleus has been studied with the ${ }^{51} \mathrm{~V}\left({ }^{10} \mathrm{~B}, \mathrm{p} 2 n\right)$ reaction at $33-\mathrm{MeV}$ incident energy and the $\gamma$-spectrometer Saci-Pererê. Excited states up to $8.0 \mathrm{MeV}$ and spin up to $11^{+}$have been observed. The results are compared to Shell Model calculations using the GXPF1 effective interaction, developed for use in the fp shell. The $\pi f_{7 / 2}^{-1} \otimes v\left(p_{3 / 2}^{2} f_{5 / 2}^{1}\right)$ configuration was assigned to the yrast levels.
\end{abstract}

Nuclei close to doubly magic shell closures are the object of extensive experimental and theoretical investigations [13]. Spectroscopic data from these nuclei provide essential information for the parameter sets of spherical shell-model calculations. They apply severe constraints on the outcome of such calculations and, consequently, define the effective nuclear forces. During recent years both experimental and theoretical efforts have been used to understand the nuclei near the $\mathrm{Z}=\mathrm{N}=28$ shell closure. There is an $\mathrm{N}$ or $\mathrm{Z}=28$ "magic" number inside the major shell with the oscillator quantum number $\mathrm{N}=3$. The shell gap at $\mathrm{N}=\mathrm{Z}=28$ is due to the spin-orbit lowering of the $f_{7 / 2}$ orbital. This gap is relatively small so that the particle-hole excitation across the gap has relatively low energies. For shell model calculations around this magic number, ${ }^{56} \mathrm{Ni}$ has often been assumed as an inert core. However, it has been shown that this core is rather soft and only a very limited description is provided by the closed-shell model for the magic number 28 [4]. These structures were successfully described for $\mathrm{N}$ or $\mathrm{Z}=28$ nuclei only after considering the existence of significant core-excitations in low-lying non yrast states as well as in high-spin yrast states [3]. These calculations were performed for the ${ }^{53} \mathrm{Mn},{ }^{54} \mathrm{Fe},{ }^{55} \mathrm{Co}$ and ${ }^{56,57,58,59} \mathrm{Ni}$ nuclei.

In this study we present new results on excited states of ${ }^{58} \mathrm{Co}$, thus enriching the systematics of the nuclear structure along the $\mathrm{N}=31$ chain. This nucleus has three particles and one hole relative to the ${ }^{56} \mathrm{Ni}$ core and has been studied so far with proton and $\alpha$ particle induced reactions [6-8], therefore very little was known regarding its high-spin structure.

The ${ }^{58}$ Co nuclei were produced with the fusion-evaporation reaction ${ }^{51} \mathrm{~V}\left({ }^{10} \mathrm{~B}, \mathrm{p} 2 \mathrm{n}\right)$ at $33 \mathrm{MeV}$ bombarding energy, with the 8MV Pelletron accelerator of the University of São Paulo (USP). The target consisted of a stack of 3 self-supporting natural ${ }^{51} \mathrm{~V}$ foils of $200 \mu \mathrm{g} / \mathrm{cm}^{2}$. Gamma-gamma-charged particle coincidences were measured with the Saci-Pererê $\gamma$ ray spectrometer. Saci [9] (Sistema Ancilar de Cintiladores) is a $4 \pi$ - charged particle system consisting of 11 plastic phoswich scintillator $\triangle \mathrm{E}-\mathrm{E}$ telescopes. Pererê [10] (Pequeno Espectrômetro de Radiação Eletromagnética com Rejeição de Espalhamento) is a $\gamma$-array spectrometer composed of $4 \mathrm{GeHP}$ detectors with BGO Compton shields (two detectors were Ortec GMX of about $20 \%$ efficiency and the other two were

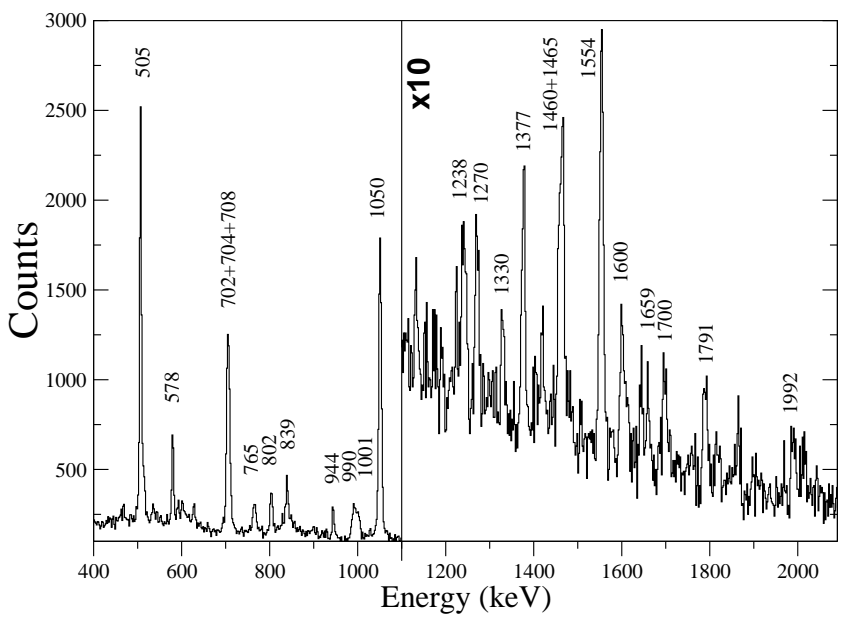

FIG. 1: Gamma-ray spectrum from the ${ }^{10} \mathrm{~B}$ on ${ }^{51} \mathrm{~V}(\mathrm{E}=33 \mathrm{MeV})$ gated on the $321 \mathrm{keV}$ low-lying transition of the ${ }^{58} \mathrm{Co}$ nucleus, and on protons detected by the SACI array. These gamma-rays were assigned to the ${ }^{58}$ Co nucleus.

Canberra REGe of $60 \%$ efficiency). Two of these detectors were placed at $37^{\circ}$ and the other two at $101^{\circ}$ with respect to the beam direction. Events were collected when at least two HPGe detectors fired in coincidence. The charged particle detectors were screened against the scattered heavy ions with three Al foils of $3.0 \mathrm{mg} / \mathrm{cm}^{2}$. A total of $48 \times 10^{6}$ Compton suppressed $\gamma-\gamma$ events was collected and registered on the hard disk of a PC. $\gamma$-ray energy and efficiency calibrations were made with ${ }^{56} \mathrm{Co},{ }^{133} \mathrm{Ba}$, and ${ }^{152} \mathrm{Eu}$ sources. The data have been Doppler corrected and sorted into symmetrized $\gamma-\gamma, \alpha$-gated, and proton-gated $\gamma-\gamma$ matrices with $9.4 \times 10^{7}$, $2.5 \times 10^{6}$ and $10.5 \times 10^{6}$ counts, respectively. Backgroundsubtracted spectra generated from those matrices were used to construct the level scheme of ${ }^{58} \mathrm{Co}$. Those matrices were analysed using the UPAK [11], GASPware [12] and Radware [13] spectrum analysis codes. The $\gamma$-ray transitions belonging to ${ }^{58} \mathrm{Co}$ were identified by setting gates on charged particle fold $1 \mathrm{p}$. In Fig. 1 the $\gamma$-ray spectrum gated on the $321 \mathrm{keV}$ low-lying transition of the ${ }^{58}$ Co nucleus, and on protons detected by the SACI array is shown. $\gamma$-rays from ${ }^{57} \mathrm{Co}$ (corres- 


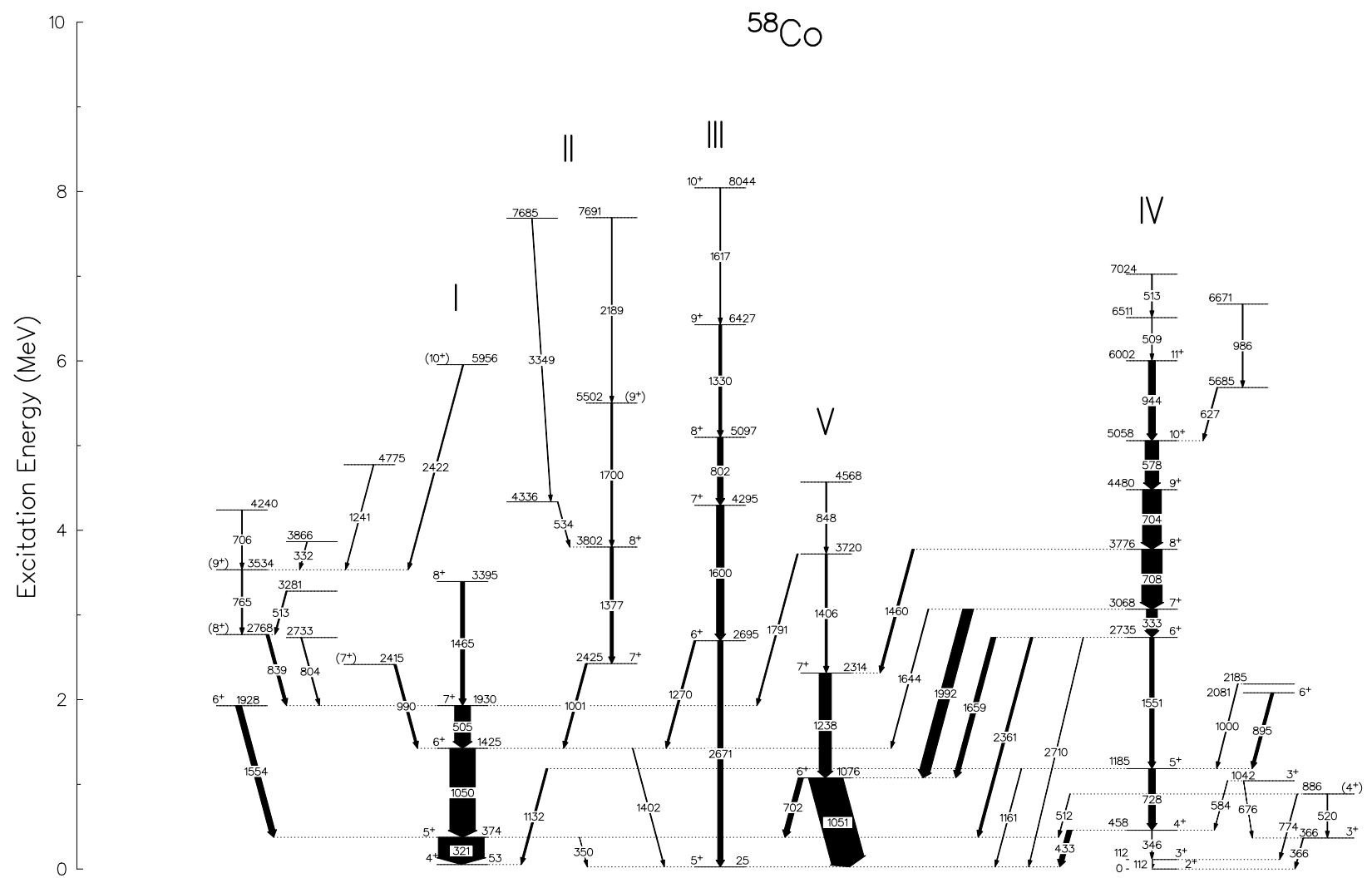

FIG. 2: The partial level scheme of ${ }^{58}$ Co obtained from the present work.

ponding to the $\mathrm{p} 3 \mathrm{n}$ channel), which is the main contaminant channel in the 1p-gated spectra, were identified from previous work [14]. The assignment of the spins to the ${ }^{58}$ Co levels was based on the DCO (directional correlation from oriented states) ratios. A $\gamma-\gamma$ matrix was constructed by sorting the data from the 2 detectors positioned at $37^{\circ}$ and $101^{\circ}$. Gates were set on both axes on several strong dipole transitions and the intensity of other transitions observed in the two spectra has been extracted. We have assumed only positive parity states since the shell model does not predict negative parity states in ${ }^{58} \mathrm{Co}$ within the excitation energy and spin limits of this work.

A level scheme extending up to an excitation energy of about $8.0 \mathrm{MeV}$ and spin $\mathrm{I}^{\pi}=11^{+}$has been proposed, based on the coincidence relationships, intensity balances on each level and energy sums from different paths using the $1 \mathrm{p}$ gated matrix (see Fig. 2). Several cascades at high excitation energy suggest a complex level structure. We have found 46 new $\gamma$-transitions de-populating 37 new excited states. The level energies are referred to the low-lying $2^{+}$state previously known [15]. The placement of the transitions in the le- vel scheme is firmly established by coincidence relationships. The width of the arrows is proportional to the intensities of transitions as seen in the reaction studied here. The relative intensities of weaker transitions, with respect to the strong ones, have been deduced from coincidence spectra.

In order to understand the observed structure, spherical shell model calculations have been performed with the code MSHELL [16]. We have used the model space and the residual interaction named GXPF1, developed recently for the description of fp-nuclei [3, 5]. The calculation with the GXPF1 interaction was performed in the full fp shell with up to 8 particle excitations from the $1 f_{7 / 2}$ orbital to the $1 p_{3 / 2}$, $1 \mathrm{f}_{5 / 2}$ and $1 \mathrm{p}_{1 / 2}$ orbitals.

To make a correspondence between a predicted level and a detected level means that both the level energies and the decay patterns should be in fair agreement. For the seven lowest yrast levels, including the g.s., the largest disagreement is for the $3^{+}$level at $112 \mathrm{keV}$ instead of the $256 \mathrm{keV}$ prediction. The theoretical calculations for the branching ratios give an idea of which transitions should be considered most impor- 


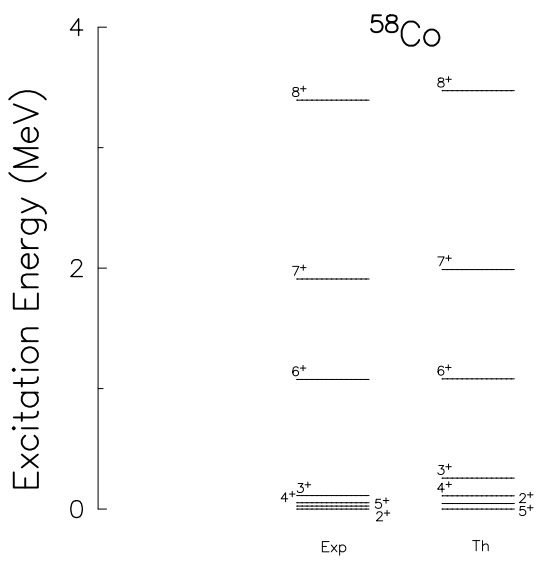

FIG. 3: Comparison between experimental and theoretical yrast excited states in ${ }^{58} \mathrm{Co}$.

tant. In Fig. 3 a comparison of the experimental level energies with the calculations for the yrast excited states of the odd-odd nucleus ${ }^{58} \mathrm{Co}$ are shown. These calculations compare reasona- bly well up to the excited yrast $8^{+}$state. The yrast states were interpreted as $\pi \mathrm{f}_{7 / 2}^{-1} \otimes \mathrm{v}\left(\mathrm{p}_{3 / 2}^{2} \mathrm{f}_{5 / 2}^{1}\right)$.

In conclusion, the level scheme of the odd-odd ${ }^{58}$ Co nucleus populated with a heavy ion fusion-evaporation reaction was measured for the first time. The shell model calculations reproduce well the experimental yrast states. Additional level assignments are being performed and should allow a more detailed comparison with the theoretical results. More experimental information on nuclei in the region of ${ }^{56} \mathrm{Ni}$ and, in particular, lifetime analysis for the excited states in ${ }^{58} \mathrm{Co}$, which are in progress, should improve the knowledge of the high spin state features.

The authors would like to thank Prof. B. A. Brown for fruitfull discussions and Prof. T. Mizusaki for providing the MSHELL calculation results. This work was partially supported by the Fundação de Amparo à Pesquisa do Estado de São Paulo (FAPESP) and the Conselho Nacional de Desenvolvimento Científico e Tecnológico (CNPq), Brazil.
[1] A. F. Lisetskiy et al., Phys. Rev. C 68, 034316 (2003).

[2] D. Rudolph et al., Eur. Phys. J. A 4, 115 (1999).

[3] M. Honma et al., Phys. Rev. C 69, 034335 (2004).

[4] T. Otsuka, M. Honma, and T. Mizusaki, Phys. Rev. Lett. 81, 1588 (1998).

[5] M. Honma et al., Phys. Rev. C 65, 061301 (2002).

[6] B. Elandsson and A. Marcinkowski, Nucl. Phys. A 146, 43 (1970) .

[7] A. C. Xenoulis and D.G. Sarantites, Nucl. Phys. A 170, 369 (1971).

[8] B. J. Brunner et al., Phys. Rev. C 11, 1042 (1975).

[9] J. A. Alcântara-Nuñez et al., Nucl. Inst. Meth. A 497, 429 (2003).
[10] R. V. Ribas et al., Ann. Rep. of the Nucl. Phys. Dep. IFUSP, 63 (1996).

[11] W. T. Milner, Holifield Heavy Ion Research Facility Computer Handbook, Oak Ridge National Laboratory, Oak Ridge, Tennessee, USA (1987).

[12] D. Bazzacco, Private comunication, INFN Sezione di Padova, Italy (1996).

[13] D. Radford, Nuclear Instrum. Methods A 361, 297 (1995).

[14] O. L. Caballero et al., Phys. Rev. C 67, 024305 (2003).

[15] M. R. Bhat, Nuclear Data Sheets 80, 789 (1997).

[16] T. Mizusaki, RIKEN Accel. Prog. Rep. 33, 14 (2000). 